Recepción: 10 / 01 / 2017

Aceptación: 16/ 06 / 2017

Publicación: 31 / 10 / 2017

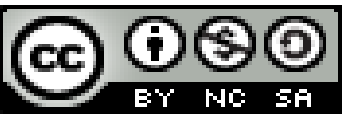

Ciencias de la salud

Artículo de investigación

\title{
Algunas técnicas alternativas al formocresol en las pulpotomías de dientes temporales
}

Some alternative techniques to formocresol in pulpotomies of temporary teeth

\author{
Algumas técnicas alternativas ao formocresol em pulpotomias de dentes \\ temporários
}

\author{
Juan A. Oliveira-del Rio ${ }^{\mathrm{I}}$ \\ juanoliveiradelrio@hotmail.com \\ Patrícia E. Bravo-Cevallos ${ }^{\mathrm{II}}$ \\ patriciabra.ce@gmail.com \\ Alba M. Mendoza-Castro ${ }^{\mathrm{III}}$ \\ lbamariamc@hotmail.com
}

Correspondencia: juanoliveiradelrio@hotmail.com

I Doctor en Ciencias Odontológicas, Universidad Laica Eloy Alfaro de Manabí, Manta, Ecuador.

II Diploma Superior en Preparación de Multirradiculares, Magister en Gerencia Educativa, Doctora En Odontología, Universidad San Gregorio de Portoviejo, Portoviejo, Ecuador.

III Odontóloga Especialista en Endodoncia, Magister en Gerencia de Salud para el Desarrollo Local, Odontóloga, Universidad Laica Eloy Alfaro de Manabí, Manta, Ecuador. 


\section{Resumen}

El objetivo principal de los tratamientos pulpares en dentición temporal, es mantener la integridad y la salud de los tejidos orales. Es deseable poder mantener la vitalidad de la pulpa de los dientes afectados por caries o traumatismos. Sin embargo, un diente puede seguir siendo funcional eliminando la pulpa parcial o totalmente. Se realizó una revisión bibliográfica con el objetivo actualizar algunas técnicas alternativas al formocresol en las pulpotomías de dientes temporales. Las técnicas con glutaraldehido, sulfato férrico y MTA son, la que los estudiosos proponen como alternativas al formocresol en las pulpotomías de dientes temporales.

Palabras clave: dientes temporales; pulpotomia; materiales y técnicas.

\section{Abstract}

The main objective of pulp treatments in temporary dentition, is to maintain the integrity and health of oral tissues. It is desirable to be able to maintain the vitality of the pulp of the teeth affected by cavities or traumatisms. However, a tooth can still be functional by removing the pulp partially or completely. A literature review was carried out with the aim of updating some alternative techniques to formocresol in pulpotomies of temporary teeth. The techniques with glutaraldehyde, ferric sulfate and MTA are the ones that the students propose as alternatives to formocresol in the pulpotomies of temporary teeth.

Keywords: temporary teeth; pulpotomy; materials and techniques.

\section{Resumo}

O principal objetivo dos tratamentos de polpa em dentição temporária é manter a integridade e a saúde dos tecidos bucais. É desejável manter a vitalidade da polpa dos dentes afetados por cavidades ou traumatismos. No entanto, um dente ainda pode ser funcional removendo a polpa parcial ou completamente. Realizou-se uma revisão da literatura com o objetivo de atualizar algumas técnicas alternativas ao formocresol em pulpotomias de dentes temporários. As técnicas com glutaraldeído, sulfato férrico e MTA são as que os alunos propõem como alternativas ao formocresol nas pulpotomias dos dentes temporários.

Palavras chave: dentes temporários; pulpotomia; materiais e técnicas. 


\section{Introducción}

En 1904 John P. Buckley desarrolló el formocresol para su empleo en la terapéutica pulpar ${ }^{1}$ y a partir de 1923 Charles A. Sweet lo utilizó para efectuar pulpotomías en dientes temporales ${ }^{2}$, desde entonces su empleo se ha generalizado hasta el punto de ser en la actualidad la técnica más utilizada en todo el mundo ${ }^{3}$, sin embargo, dados los posibles problemas de toxicidad del formocresol ${ }^{3}$ otras técnicas se han venido desarrollando en los últimos años, como alternativas a las pulpotomías con formocresol en dientes temporales ${ }^{4}$.

\section{Desarrollo}

La caries, aún en la actualidad, continúa siendo la enfermedad infecciosa transmisible no autolimitante más común en la infancia. La menor mineralización y el menor espesor de los tejidos dentarios en piezas deciduas, hacen que su avance sea rápido demandando con frecuencia tratamientos pulpares 5 .

Hoy en día existe una gran importancia por conservar los dientes naturales, muchos dientes se pierden a muy temprana edad, esta pérdida conduce a maloclusiones o a problemas estéticos y funcionales que pueden ser de manera transitoria o permanente. El objetivo fundamental de la terapia pulpar es conservar la integridad y la salud de los tejidos orales. La conservación de los dientes temporales afectados por caries profundas es motivo de gran preocupación en Odontopediatría 5 .

El gran tamaño de la cámara pulpar en dientes primarios los hace fácilmente susceptibles a la exposición por caries. La pulpotomía es uno de los tratamientos más utilizados, para mantener molares deciduo con compromiso de caries, pulpitis reversibles asintomáticas. Su objetivo es preservar la pulpa radicular, evitar el dolor, la inflamación y, mantener el diente Es un procedimiento que involucra la amputación de la porción coronal de la pulpa dental afectada o infectada y el tratamiento de los muñones radiculares con un medicamento, con el fin de que la pieza pueda preservar su vitalidad y función. La pulpa dental como todo tejido especializado cumple con funciones específicas, según las células que lo componen: formativa, sensorial, nutritiva y defensiva o protectora ${ }^{5}$. 
El objetivo principal de los tratamientos pulpares en dentición temporal, es mantener la integridad y la salud de los tejidos orales. Es deseable poder mantener la vitalidad de la pulpa de los dientes afectados por caries o traumatismos. Sin embargo, un diente puede seguir siendo funcional eliminando la pulpa parcial o totalmente ${ }^{6}$.

Las indicaciones, objetivos y el tratamiento pulpar indicado se basan en un diagnóstico clínico que determine el estado de la pulpa. Un examen preoperatorio completo es esencial para obtener un diagnóstico correcto y poder establecer el tratamiento adecuado, así como orientar en el pronóstico de éste ${ }^{6}$.

La técnica de pulpotomía consiste básicamente en la extirpación de la pulpa coronal y la colocación en la entrada de los canales de diferentes materiales, que dan el nombre al tipo de pulpotomía (pulpotomía al formocresol, pulpotomía al glutaraldehído, pulpotomía al hidróxido de calcio, etc ${ }^{7}$.

Técnica secuencial: anestesia del diente a tratar. Aislamiento absoluto y esterilización del campo operatorio. Eliminación completa del tejido cariado. Apertura de la cámara pulpar a través de la exposición, entrando en cámara con fresa redonda $\left(n^{\circ} 6\right.$ al $\left.n^{0} 11\right)$ con contra ángulo a baja velocidad. Eliminación del techo cameral, en su caso, aplicando la fresa desde dentro de la cámara contra el techo y haciéndola girar impulsándola hacia fuera. No debe quedar dentina sobresaliente en el techo de la cámara pulpar o en los cuernos pulpares. Eliminación del tejido pulpar cameral con una cucharilla discoide afilada o un excavador, hasta la entrada de los conductos radiculares. Los muñones de la pulpa a nivel de los orificios de entrada de los conductos radiculares serán muy bien cortados de forma que no queden restos de tejido pulpar en el suelo de la cámara. Lavado de la cámara pulpar con jeringa y bolitas de algodón impregnadas en agua de cal, suero fisiológico o agua oxigenada diluida, hasta eliminar los restos pulpares y conseguir cohibir la hemorragia. Debe comprobarse que se han formado coágulos a la entrada de los conductos radiculares. Aplicación de la pasta de hidróxido de calcio puro sobre el tejido pulpar remanente de los conductos, colocando con un portaamalgamas una porción de la pasta sobre la entrada de cada uno de los conductos, presionando ligeramente con una bolita de algodón y procurando que dicha entrada quede bien sellada. Aplicación de una capa de óxido de 
zinc - eugenol (IRM) de al menos $1 \mathrm{~mm}$ de espesor. Restauración final del diente según la conveniencia o la planificación previamente realizada ${ }^{7}$.

\section{Observación y pronóstico}

Se harán revisiones a los 3 y a los 6 meses para comprobar la ausencia de signos clínicos y radiológicos, y que el desarrollo radicular prosigue con normalidad, pudiéndose afirmar que se ha obtenido el éxito cuando se observa en la radiografía la formación de un "puente dentinario" que separa la cámara pulpar de cada conducto radicular. Es muy útil comparar la imagen radiológica del diente tratado con la del diente contralateral para valorar adecuadamente el desarrollo radicular ${ }^{7}$.

\section{Técnicas y materiales}

\section{Óxido de cinc-eugenol}

El óxido de cinc-eugenol se utiliza ampliamente como una base en las pulpotomías, debido a sus propiedades antibacterianas y analgésicas. Además, proporciona un sellado eficaz, lo que limita la microfiltración e infección recurrente posterior. Sin embargo, la colocación directa del eugenol sobre el tejido pulpar puede dañarlo, pero si el tejido pulpar es fijado con un agente como puede ser el formocresol, el tejido pulpar no va a ser afectado por el eugenol ${ }^{8}$.

Algunos autores han estudiado la aplicación directa del óxido de cinc-eugenol sobre los muñones pulpares, obteniendo unos resultados similares a los del formocresol. Estos autores atribuyen más el éxito a la remoción mecánica de la pulpa coronal inflamada que a las propiedades curativas del medicamento utilizado ${ }^{8}$.

\section{Hidróxido de calcio}

Los procedimientos de antisepsia en endodoncia, cobran una significación especial, la ausencia de gérmenes tiene una importancia capital en el buen resultado de los tratamientos, realizados en el campo de esta especialidad. Numerosos han sido los medicamentos que se han empleado con este fin. Dentro de esta amplia gama de agentes se encuentra el hidróxido de calcio $(\mathrm{CaOH})$, cuyo uso en odontología ha despertado recientemente un interés considerable. 
Luego de haber seguido los pasos previos, después de la hemostasia, aplicamos una torunda de algodón impregnada con hidróxido de calcio y se espera a que actué el medicamento en los muñones y se coloca una capa de hidróxido de calcio sobre ellos se espera a que sequé y se coloca un cemento a base de óxido de zinc y eugenol. A pesar de su amplia aplicación, las bajas tasas de éxito en el recubrimiento pulpar y pulpotomías han limitado el uso de $\mathrm{Ca}(\mathrm{OH}) 2$ como un apósito en pulpotomías en dentición decidua. Investigaciones han demostrado que el uso de $\mathrm{Ca}(\mathrm{OH}) 2$ da lugar con frecuencia a un desarrollo de inflamación crónica de la pulpa y la incidencia reabsorción radicular. La desventaja principal de este material es la reabsorción interna, que, como se pensaba, era estimulada por dicho material. Esta reabsorción es producida por un coagulo de sangre que interviene entre el material y el tejido pulpar ${ }^{5}$.

En 1962 se comparó la técnica de la pulpotomía con formocresol, con la técnica del hidróxido de calcio en caninos deciduos, y se encontró que el formocresol producía un éxito clínico del 95\% al término de un año. En el examen histológico, aunque se observó fijación del tejido pulpar y algo de pérdida de la definición celular, fue evidente el tejido sano y vital en el tercio apical. La técnica del hidróxido de calcio se consideró con un éxito del 61\%, observándose la formación de puentes de dentina en el $50 \%$ de los casos examinados ${ }^{5}$.

Pese a sus aplicaciones en la capa pulpar o procedimientos de pulpotomia, el hidróxido de calcio no es generalmente preferido en recubrimiento pulpar en dientes primarios debido al limitado éxito clínico.

El análisis crítico de la literatura sugiere, sin embargo, que los resultados de la pulpotomia pueden ser afectados significativamente como variable en la técnica, en uso en calidad de materiales, y la restauración final.

El porcentaje de éxito clínico y radiológico, disminuye con el tiempo de una manera muy marcada. También se encuentran los datos sobre reabsorciones internas, dado que con esta técnica son muy frecuentes y suponen una de las principales causas de fracaso. Las reabsorciones internas tienden a aumentar a medida que pasa el tiempo. Hay que destacar que apenas hay datos sobre obliteración interna o metamorfosis cálcica ni sobre alteraciones sobre el diente permanente sucesor, posiblemente porque no se dan con esta técnica ${ }^{4}$. 
Se realizó un estudio con la finalidad de determinar el éxito, en pulpotomia con hidróxido de calcio y curaciones con ionomero de vidrio como material de sellaje de la pulpa en, aproximadamente 2 años. El éxito de los resultados se compara con los estudios en el cual se usaron el material de sellaje con óxido de zinc y eugenol. El estudio demostró que la mezcla de agua y el hidróxido de calcio, cumplen los requerimientos de una suspensión que debe ser usada como un recubridor'.

\section{Electrocoagulación}

La técnica sigue la pauta ya comentada, se aplica una descarga de corriente eléctrica, durante 1 ó 2 segundos, con el electrodo colocado a una distancia de 1-2 milímetros del muñón amputado y repitiendo la operación en todos los muños a intervalos de 5-10 segundos para evitar el excesivo calentamiento de la cámara pulpar. La maniobra se repite 2-3 veces por cada muñón, colocando en cada intervalo una bolita de algodón, para que absorba cualquier resto de sangre o fluidos antes de aplicar la corriente eléctrica. Los electrobisturís que se han empleado hasta la fecha son el Hyfrecator 705A y el Storbex Ultron, siempre a media potencia. Finalmente se rellena la cámara pulpar con un cemento tipo IRM, óxido de zinc eugenol, oxifosfato de zinc, como en el caso anterior ${ }^{4}$.

Los resultados de cuatro estudios clínicos que abarcan un tiempo de controles entre los seis meses a los dos años, son sorprendentes puesto que, en los estudios con controles a más largo plazo, los resultados clínicos y radiológicos mejoran en contra de la lógica de mantenerse o disminuir. La obliteración de conductos sólo aparece en uno de los estudios a seis meses y no hay datos de alteración en el sucesor permanente ${ }^{4}$.

\section{Glutaraldehído}

El glutaraldehído es una alternativa química, que ha sido propuesta para el tratamiento de las pulpotomías en dientes primarios, y ha recibido particular atención como sustituto del formocresol, porque es un fijador suave y potencialmente menos toxico. Además, es un potente antiséptico y antibacteriano, con una molécula muy grande, lo cual hace que su distribución sistémica sea más limitada cuando se comprara con el formocresol ${ }^{5}$. 
Se prepara una bolita de algodón estéril embebida en glutaraldehído al 2\%, previamente exprimida antes de colocar en la entrada de los conductos radiculares, se espera 5 minutos y luego se puede proceder a restaurar la pieza dentaria ${ }^{5}$.

En los resultados destaca como el porcentaje de éxito clínico y radiológico, tiende a disminuir con el tiempo, pero aun así alcanza unos resultados bastante buenos. Por otra parte, la metamorfosis cálcica también tiende a aumentar con el tiempo. Sobre el sucesor permanente encontramos que la erupción se encuentra adelantada en el 15\% de los casos y estos no presentan defectos del esmalte ${ }^{4}$.

\section{Sulfato férrico}

El sulfato férrico se ha utilizado al 15,5\% (Astringedent) y actualmente al 20\% (Viscostat). Este compuesto de hierro se utiliza por su acción fuertemente hemostática y su efecto bactericida moderado, pero no tiene acción fijadora de tejidos o momificante ${ }^{10}$.

La técnica sigue la pauta ya comentada, Tras limpiar de restos de sangre la cámara pulpar y observar que apenas sangran los muñones se aplica el Viscostat, en unas jeringas especiales y con unas boquillas metálicas con punta de cepillo (dentoinfusor), apretando sobre cada uno de los muñones radiculares del suelo de la cámara pulpar, presionado sobre cada uno unos 10-15 segundos. Posteriormente se limpia con el chorro de agua y se seca quedando la cámara de color amarillo-marrón. Si tras la aplicación sangra algún muñón, se puede volver a aplicar. Como en todas las pulpotomías se rellena la cámara con cemento (IRM, OZE, fosfato de zinc...) ${ }^{10}$.

Recientemente, Ibricevic y cols, publicaron un estudio clínico en humanos, comparando el uso de sulfato férrico y de formocresol en pulpotomías de dientes temporales. Se analizaron 35 molares en cada grupo, con un tiempo máximo de estudio de 20 meses. Los resultados mostraron un éxito clínico valorado en un $100 \%$ y un éxito radiográfico de 97,2\% en ambos grupos, hallándose en $2,8 \%$ de los casos reabsorciones internas. Los autores concluyeron que, ante la igualdad de resultados, y dado que el sulfato férrico no es tóxico y es fácil de manipular. ${ }^{11}$

\section{Mineral Trióxido Agregado (MTA)}

El MTA es un cemento muy prometedor en el campo de la endodoncia. Fue autorizado por la Food and Drug Administration (Departamento del Ministerio de Sanidad de los Estados Unidos) 
en 1998 y comenzó a utilizarse en pulpotomías de dientes temporales en 2001. El preparado que se emplea es el gris dado que el blanco tiene peores resultados. ${ }^{4}$

La técnica es como hemos descrito en los casos anteriores y una vez que se ha eliminado la pulpa cameral y controlada la hemorragia se prepara el cemento MTA. El polvo gris de MTA se mezcla con suero salino en una proporción de tres partes de polvo por una de líquido. Una vez mezclado se lleva a la cámara pulpar con una porta amalgamas y se adapta al suelo con la presión de una bolita de algodón húmeda. Los restos se eliminan de las paredes y se rellena el resto de la cámara pulpar con un cemento tipo IRM, óxido de zinc eugenol, oxifosfato de zinc, como en los casos anteriores. ${ }^{4}$

Los resultados son excelentes tanto clínicos como radiológicos, puesto que tienen éxito el 100\% de los casos y no hay disminución con el tiempo. También se observa que con el paso del tiempo si tiende a aumentar las obliteraciones de los conductos radiculares. No hay datos de alteración en los dientes sucesores permanentes. ${ }^{4}$

Según la literatura revisada es posible afirmar que, en general, el MTA es considerado una excelente alternativa al formocresol, para las pulpotomías en molares deciduas. Sin embargo, la mayoría de los autores consideran que este material presenta algunas desventajas. Indudablemente, el precio elevado del MTA es el factor más frecuentemente apuntado, como limitación a la utilización de este material en la práctica clínica diaria. El tiempo de fraguado, de 3 a 4 horas, también es considerado por algunos autores como una desventaja, principalmente cuando se compara con el tiempo necesario para la conclusión del tratamiento en el caso de utilización del formocresol ${ }^{12}$.

Para concluir, las técnicas con glutaraldehido, sulfato férrico y MTA son, la que los estudiosos proponen como alternativas al formocresol en las pulpotomías de dientes temporales. 


\section{Referencias Bibliográficas}

1- Buckley jp. A rational treatment for putrescent pulps. Dent Rev 1904; 18: 1193-7

2- Sweet ca. Root canal treatment in deciduous teeth, incluiding pulp exposure. Pacific Dent Gaz 1923;31:718-21

3- Avram dc, pulver f. Pulpotomy medicaments for vital primary teeth. Surveys to determine and attitudes in pediatric dental practice and in dental school throught the world. J Dent Child 1989; 56(6):426-34

4- Calatayud J, Casado I, Álvarez C. Análisis de los estudios clínicos sobre la eficacia de las técnicas alternativas al formocresol en las pulpotomías de dientes temporales. Av Odontoestomatol. 2006 [citado 2018 Ene 09]; 22(4): 229-239. Disponible en: http://scielo.isciii.es/scielo.php?script=sci_arttext\&pid=S0213$12852006000400004 \& \operatorname{lng}=\mathrm{es}$

5- García Solís A L. Pulpotomías en dientes deciduos: materiales y técnicas"2011. Investigación bibliográfica del proceso de suficiencia Profesional para obtener el título de cirujano dentista. [citado 2018 Ene 09]. Disponible en: www.cop.org.pe/bib/investigacionbibliografica/AYLINLORELEYGARCIASOLIS.pdf

6- Protocolo para los tratamientos pulpares en dentición temporal. 2014. [citado 2018 Ene 09]. Disponible en: http://www.odontologiapediatrica.com/pulpa

7- Guillén Chujutalli T R .Avances de terapia pulpar en odontología pediátrica. 2008 [citado Ene 09]. 2018 Disponible www.cop.org.pe/.../investigacionbibliografica/TEDDYRODOLFOGUILLENCHUJUT

8- Fernández Delgado FJ, López Trujillo JM, Vallejo Bolaños E. Alternativas a la pulpotomía con formocresol en odontopediatría. Quintessence. 2001; 14: 385-92

9- Rene J. M. Pulpotomia con Hidróxido de calcio con material sellaje en cavidades curadas en 2 años. 1999 [citado 2018 Ene 09]. Disponible en: sisbib.unmsm.edu.pe/BVRevistas/odontologia/1999_n4/pdf/pulpotomia.pdf 
10- Fei a-1, udin rd, johnson r. A clinical study of ferric sulfate as a pulpotomy agent in primary teeth. Pediatr Dent 1991;13(6): 327-32

11- Ibriamecevic H, Al Jame. Ferric Sulfate as pulpotomy agent in primary teeth. J Clin Pediatr Dent. 2000;24(4):269-72

12- Bellet1 L, Guinot F, Arregui M. Aplicaciones clínicas del MTA en odontopediatría. Dentum 2006;6(3):96-102 